\title{
Effects of Hydrogen Bonds on the Mobility of Spin Probes in Nylon Films
}

\author{
Kunihiro HAMAdA, Toshiro IIJIMA, and Ralph MCGREgOR* \\ Department of Polymer Science, Tokyo Institute of Technology, \\ Ookayama, Meguro-ku, Tokyo 152, Japan \\ *Department of Textile Chemistry, North Carolina State University, \\ Raleigh, North Carolina 27695-8302, U.S.A.
}

(Received December 23, 1986)

\begin{abstract}
To elucidate the effects of hydrogen bonds between polymer chains and small molecules on the mobility of small molecules, the mobilities of TEMPO (SPIV), TEMPOL containing a hydroxyl group (SPV), and TEMPONE containing a carbonyl group (SPVI) were investigated by means of electron spin resonance (ESR) measurements, and compared with the mobility of TEMPAMINE containing amino group (SPIII) examined previously. $T_{50 \mathrm{G}}$, at which the extrema separation of ESR spectra becomes $5 \mathrm{mT}(50 \mathrm{G})$ decreased in the order of SPVI $>$ SPIII $>$ SPV $>$ SPIV, suggesting that the functional groups of the spin probes strongly affect the mobilities of the spin probes. For all the spin probes, $T_{50 \mathrm{G}}$ decreased with an increase in the methylene chain length of the nylon. However, the effects of drawing on the mobility were found only for SPIII. In the Arrhenius plots of rotational correlation times, one or two crossover points were defined. The crossover point at the lower temperature, $T_{\mathrm{n}}{ }^{\prime}$, is considered to be the temperature at which the full rotation of a piperidine ring in the spin probes occurs, and that at the higher temperature, $T_{n}$, is presumed to be the temperature at which the rotational motion of the whole probe molecule becomes coupled with translational diffusion. For SPVI only, could $T_{\mathrm{n}}{ }^{\prime}$ not be observed. The variation of the rotational behavior in the spin probes is due to the way in which hydrogen bonds form between their functional groups and the nylon chains. Such a variation was also found in the activation energies for rotation. The activation energy for rotation in the temperature region between $T_{\mathrm{n}}{ }^{\prime}$ and $T_{\mathrm{n}}$ was greatly dependent on the structure of the spin probe.

KEY WORDS Spin Probe / ESR / Nylon / Nitroxide / Hydrogen Bond / Molecular Motion /
\end{abstract}

It is well-known that the permeability of penetrants is dependent on their size. For solute permeation through membranes, the effects of the size on the permeability have been discussed by means of free volume theories, ${ }^{1,2}$ e.g., Wisniewski et al. explained the effects of apparent solute molecular size on the diffusion coefficient in poly(2-hydroxyethyl methacrylate) by using the free volume theory, ${ }^{3}$ and Peppas et al. developed a new theoretical model based on the free volume theory to correlate the solute diffusion coefficient with the solute size. ${ }^{4,5}$ In addition, for the membrane transport of alkali cations and halide anions, the effects of their size have been discussed. ${ }^{6}$ However, the fixation of crown ethers to the membrane makes it possible to change the permeability of a particular alkali cation. ${ }^{7}$ In such a case, another factor, i.e., the interaction between the crown ethers and the cations must be considered. Thus the interaction between the penetrant molecule and the polymer matrix plays an important role in membrane transport.

On the other hand, the effects of probe molecular size on the rotational motions of spin probes have been investigated. ${ }^{8-10}$ In these studies, the segmental volume concerned 
in the molecular motion of the polymer is related to the molecular volume of the spin probe by using Bueche's free volume theory. ${ }^{11}$ As some authors have pointed out, however, the interactions between polymers and probe molecules, ${ }^{9}$ and the free rotation around the single bonds in some probes ${ }^{10}$ make the use of this simple relation inappropriate. In our previous papers, ${ }^{12,13}$ we reported on the mobilities of three nonionic spin probes having different molecular volumes, in nylon films. It was shown that the mobilities of the spin probes were influenced not only by the molecular volumes of the spin probes, but also by the interactions between the probe molecules and the nylon chains. Also the mobilities of anionic spin probes in the nylon films reflected the mobility of the amino end groups of the nylons through electrostatic interactions. ${ }^{14,15}$ It was thus demonstrated that the interactions between probe molecules and polymer chains should not be ignored for the nylon films.

In the present study, to elucidate the effects of the interactions on the mobilities of spin probes, the mobilities of spin probes having different functional groups with similar molecular volumes was investigated by means of electron spin resonance (ESR) measurements. The effects of the functional groups of the spin probes are discussed in detail, on the basis of the change of the mobility with methylene chain length, and on drawing.

\section{EXPERIMENTAL}

To compare with 4-amino-2,2,6,6-tetramethyl-1-piperidinyloxy (TEMPAMINE, SPIII), which was investigated in our previous paper, ${ }^{13}$ three nonionic spin probes, 2,2,6,6tetramethyl-1-piperidinyloxy (TEMPO, SPIV), 4-hydroxy-2,2,6,6-tetramethyl-1-piperidinyloxy (TEMPOL, SPV), and 4-oxo-2,2,6,6tetramethyl-1-piperidinyloxy (TEMPONE, SPVI) were used. SPVI was purchased from Aldrich Co., SPV and SPVI from Eastman Kodak Co., and they were used without further purification.

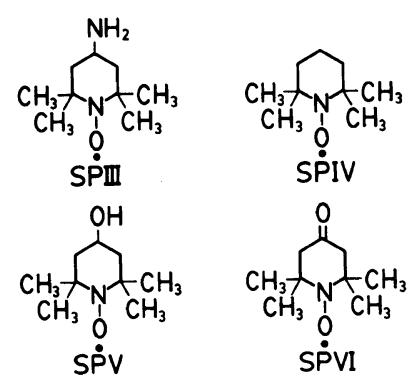

Five nylon films were used. Undrawn nylon 4 film (NY-4) was prepared as described in our previous papers. ${ }^{13,15}$ Biaxially drawn nylon 6 film (NY-6(2D)) and undrawn nylon 6 film (NY-6(ND)) were kindly supplied by Unitika Co. Nylon 11 film (NY-11) and nylon 12 film (NY-12), both drawn by inflation, were cordially supplied by Daicell Co. The nylon films were pretreated in boiling water as reported in our previous paper. ${ }^{13}$

The spin probes were sorbed by the nylon films from the aqueous probe solutions at $343 \mathrm{~K}$ for 2 days. The amount of the spin probes sorbed were about $5 \times 10^{-6} \mathrm{~mol} \mathrm{~g}^{-1}$ of the nylon. After sorption, the film was put into an ESR tube, dried in vacuo at $393 \mathrm{~K}$ for 1 day, and sealed in the presence of air. ESR spectra were measured under the same conditions as described in our previous papers. $^{12-14}$

\section{RESULTS AND DISCUSSION}

\section{Extrema Separation}

The separation of the outer extrema of the ESR spectra, $2 A z^{\prime}$, which is a measure of the mobility of the spin probe, decreases with increasing mobility (see Figure 2 of ref 13). As shown in Figures 1 and 2, $2 A z^{\prime}$ decreased with increasing temperature in all the systems, indicating that the mobilities of the spin probes increased with an increase in temperature. For all the spin probes, $2 A z^{\prime}$ decreased with increasing methylene chain length of the nylons, i.e., the increase of the methylene chain length 


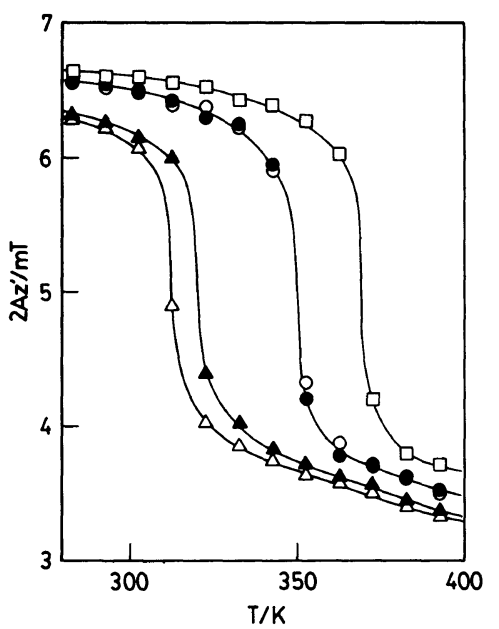

Figure 1. $2 A z^{\prime}$ vs. temperature for $\mathrm{SPV}(-\mathrm{OH})$. $\square, \mathrm{NY}-$ 4;, $\mathrm{NY}-6(2 \mathrm{D})$; O, NY-6(ND); $\Delta, \mathrm{NY}-11 ; \triangle, \mathrm{NY}-12$.

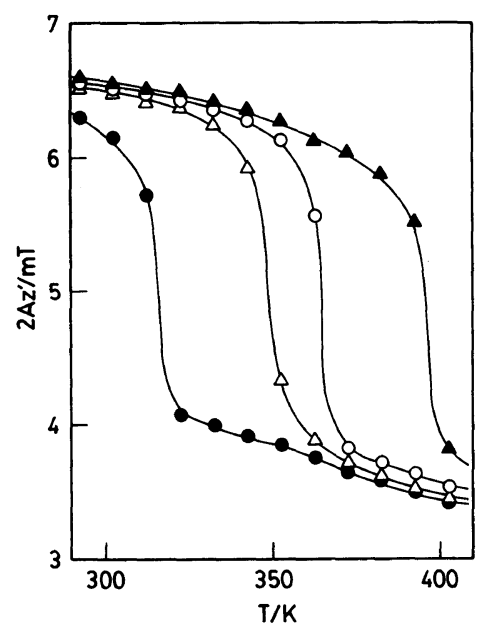

Figure 2. $2 A z^{\prime} v s$. temperature for NY-6(ND). $\bigcirc$, SPIII* $\left(-\mathrm{NH}_{2}\right) ; 0, \operatorname{SPIV}(N o n e) ; \triangle, \operatorname{SPV}(-\mathrm{OH}) ; \boldsymbol{\Delta}$, $\operatorname{SPVI}(=0) .{ }^{*} C f$. ref 13 .

resulted in an increase of the mobility of the probe molecules. However, no effect of drawing was observed for SPIV, SPV, and SPVI. On the other hand, the $2 A z^{\prime}$ values for SPIII in the drawn film were very different from those in the undrawn film. ${ }^{13}$ This is thought to be explained as follows. The amino group of SPIII can form crosslinkage between the nylon chains through two hydrogen bonds (Figure $3 \mathrm{a})$, and higher orientation of the nylon chains (a)

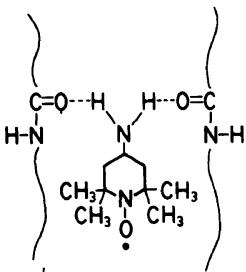

(b)

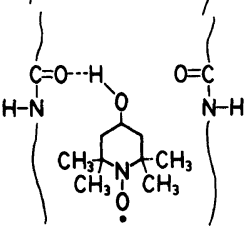

(c)

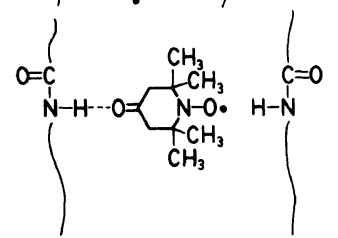

Figure 3. Model of the interaction between the spin probes and the nylon chains.

makes it easier to form this crosslinkage. In the undrawn film the crosslinkage between the nylon chains through two hydrogen bonds is hardly formed, so that SPIII probably rotates less restrictedly than the drawn film.

The change of $2 A z^{\prime}$ with temperature varied from probe to probe (Figure 2). Here it is convenient to use $T_{50 \mathrm{G}}$, the temperature at which $2 A z^{\prime}$ becomes $5 \mathrm{mT}(50 \mathrm{G})$, for discussion (Table I). $T_{50 \mathrm{G}}$ decreased in the order of SPIII-SPVI $>$ SPV $>$ SPIV for all the films except NY-6(ND). In the case of NY-6(ND), $T_{50 \mathrm{G}}$ for SPVI was much larger than that for SPIII. The lower $T_{50 \mathrm{G}}$ value for SPIII in NY-6(ND) is probably due to the specific behavior of the amino group of SPIII as mentioned above. The difference of $T_{50 \mathrm{G}}$ in the spin probes may be explained by considering the hydrogen bonding between the probe molecules and the nylon chains, and the rotation around a single bond in the spin probes. SPV and SPVI have a hydroxyl and a carbonyl group, respectively, so that hydrogen bonds between these functional groups and the nylon chains can be formed (Figures $3 b$ and 3c). SPVI has no single bond around which to 
Table I. $T_{50 \mathrm{G}}(\mathrm{K})$

\begin{tabular}{lcccccc}
\hline & Substituent & NY-4 & NY-6(2D) & NY-6(ND) & NY-11 & NY-12 \\
\hline SPIII $^{\mathrm{a}}$ & $-\mathrm{NH}_{2}$ & 416 & 391 & 365 & 360 & 354 \\
SPIV & None & 340 & 316 & 317 & 260 & 255 \\
SPV & $-\mathrm{OH}$ & 370 & 350 & 350 & 320 & 313 \\
SPVI & $=\mathrm{O}$ & 409 & 396 & 397 & 361 & 351 \\
\hline
\end{tabular}

a $C f$. ref 13 .

rotate, resulting in a stronger restriction, i.e., a higher $T_{50 \mathrm{G}}$ value. SPV has single bonds around which to rotate, and this rotation is thought to decrease $T_{50 \mathrm{G}}$. Naturally the rotation around the hydrogen bonds should be taken into consideration, but from these results only, we can not reach definite conclusions. On the other hand, SPIV has no additional functional group with which to form hydrogen bonds, leading to the lowest $T_{50 \mathrm{G}}$ value.

For all the spin probes, $T_{50 \mathrm{G}}$ decreased with increasing methylene chain length. The difference between $T_{50 \mathrm{G}}$ for NY-4 and NY-12, was almost the same for SPIII $(62 \mathrm{~K})$, SPV $(57 \mathrm{~K})$, and SPVI $(58 \mathrm{~K})$, while that for SPIV (85K) was much larger than the former values, suggesting that the spin probes forming no hydrogen bond monitors the mobility of the hydrophobic regions of the nylon chains more clearly. Effects of drawing on $T_{50 \mathrm{G}}$ were not found for SPIV, SPV, and SPVI, but were observed only for SPIII. A possible reason for this has already been mentioned.

$T_{50 \mathrm{G}}$ has been empirically correlated with the glass transition temperature $T_{\mathrm{g}} \cdot{ }^{8,9}$ In these empirical correlations between $T_{50 \mathrm{G}}$ and $T_{\mathrm{g}}$, only the molecular volumes of the spin probes were taken into account, i.e., $T_{50 \mathrm{G}}$ increases with increasing molecular volume of the probe molecule. However, the molecular volumes of the spin probes used in the present study are similar, so that the difference in $T_{50 \mathrm{G}}$ of the probe molecules can not be explained only by the molecular volumes. Thus the empirical correlations between $T_{50 \mathrm{G}}$ and $T_{\mathrm{g}}$ can not be used for the systems where probe molecules interact with polymers.

\section{Rotational Correlation Times}

For further discussion, rotational correlation times $\tau_{\mathbf{R}}$ were determined from the ESR spectra. As was reported in our previous papers, ${ }^{12,13}$ the equations derived by Kivelson ${ }^{16}$ and Kuznetsov et al. ${ }^{17}$ were used for the calculation of $\tau_{R} \cdot \tau_{R}$ used here was determined with the assumption of isotropic rotation, and it should be termed the "apparent rotational correlation time," so that it was used for the purpose of comparison.

The Arrhenius plots of $\tau_{R}$ are shown in Figures 4 and 5 . Under the assumption that the plot is divided into two or three straight lines, the calculation for the individual cases is repeated by the least squares method until a correlation coefficient in each region is greater than 0.98 , as described in our previous paper. ${ }^{15}$ Although $\tau_{\mathrm{R}}$ values below $283 \mathrm{~K}$ were not shown in Figure 5, all experimental points below $283 \mathrm{~K}$ lie on the straight lines in the lowest temperature region. For SPIV and SPV, two distinct crossover points were defined, but only one was defined for SPVI. As mentioned in our previous paper, ${ }^{13}$ SPIII had a different number of crossover points in different nylons, i.e., NY-4 and NY-6(2D) had only one, while NY-6(ND), NY-11, and NY-12 had two distinct crossover points in the temperature range investigated. This was explained as follows. ${ }^{13}$ In the case of NY-4, the second crossover point exists in a higher temperaure region than 


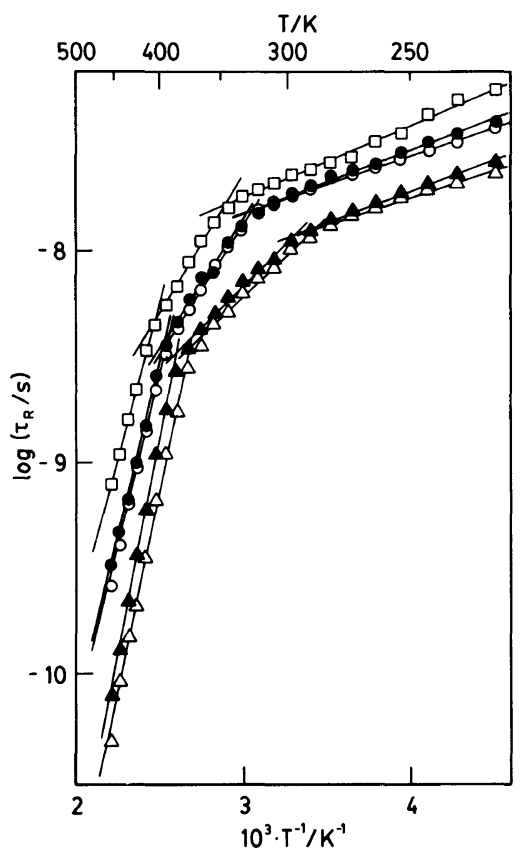

Figure 4. Arrhenius plots of $\tau_{\mathrm{R}}$ for $\operatorname{SPV}(-\mathrm{OH}) . \square$, NY-4; O, NY-6(2D); O, NY-6(ND); $\Delta$, NY-11; $\triangle$, NY-12.

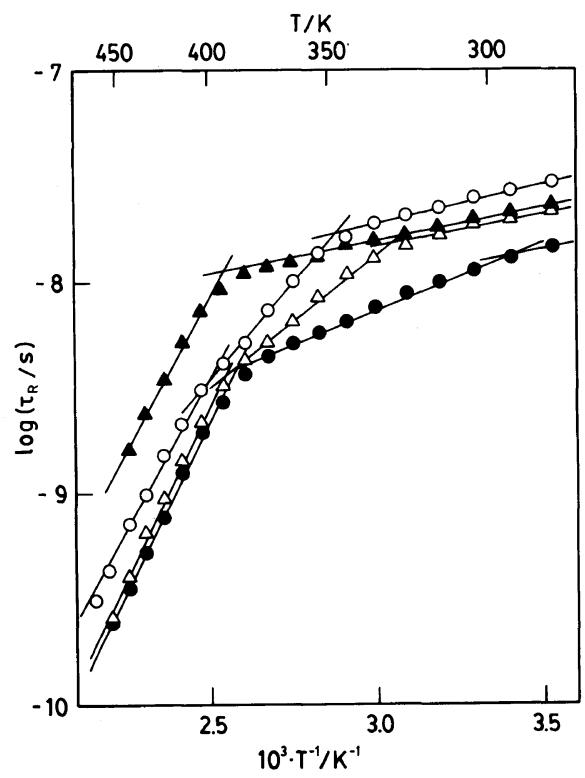

Figure 5. Arrhenius plots of $\tau_{R}$ for NY-6(ND). $O$, SPIII* $\left(-\mathrm{NH}_{2}\right) ; 0, \operatorname{SPIV}(\mathrm{None}) ; \triangle, \operatorname{SPV}(-\mathrm{OH}) ;$ $\operatorname{SPVI}(=0) .{ }^{*} C f$. ref 13 . the temperature range examined. For NY6(2D), the slopes of the Arrhenius plots below and above the second crossover point were presumably quite similar, and consequently we could not find it. It is thus concluded that SPIII, SPIV, and SPV have two distinct crossover points, while SPVI has only one.

The crossover point in the low temperature region for SPIII, SPIV, and SPV is designated as $T_{\mathrm{n}}{ }^{\prime}$ and that in the high temperature region as $T_{\mathrm{n}}$. The crossover point for SPVI is also designated as $T_{\mathrm{n}}$. The existence of these crossover points may be explained as follows. For SPIII, $T_{\mathrm{n}}{ }^{\prime}$ is thought to be the temperature at which the rotation around a single bond in the probe molecule occurs, because SPIII is bound to the nylon chains through hydrogen bonds. ${ }^{12,13} T_{\mathrm{n}}{ }^{\prime}$ for SPV is interpreted in the same way. However, SPIV can form hydrogen bonds only with difficulty, and has no single bond around which to rotate, so that $T_{\mathrm{n}}{ }^{\prime}$ for SPIV can not be explained in a similar way to SPIII and SPV. Full rotation of SPIV without translational diffusion probably occurs at $T_{\mathrm{n}}{ }^{\prime}$. The other probe molecules have the possibility of such full rotation, but the restriction through the hydrogen bonds prevents them from rotating fully. Also the appearance of $T_{\mathrm{n}}{ }^{\prime}$ for the spin probes which interact weakly with the nylon chains is probably dependent on their molecular volumes. For the larger spin probe, SPII, which it is thought can hardly interact with the nylon chains, $T_{\mathrm{n}}{ }^{\prime}$ could not be observed. ${ }^{13}$

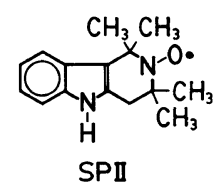

Since SPVI is strongly restricted by the nylon chains, and has no single bond around which to rotate, it is thought that $T_{\mathrm{n}}{ }^{\prime}$ did not appear. Here it is worthwhile to consider again the possibility of rotation around the hydrogen bonds. If this rotation occurs, $T_{\mathrm{n}}{ }^{\prime}$ would be 
observed even for SPVI. The fact that $T_{\mathrm{n}}{ }^{\prime}$ did not appear for SPVI suggests that this rotation scarcely takes place. It is thus concluded that the existence of $T_{\mathrm{n}}{ }^{\prime}$ is dependent on the interaction between the spin probes and the nylon chains, the existence of single bonds around which to rotate, and the molecular volumes of the spin probes. On the other hand, $T_{\mathrm{n}}$ is believed to be the temperature at which the rotational motion of the whole probe molecule occurs with translational diffusion. ${ }^{13}$ $T_{\mathrm{n}}{ }^{\prime}$ and $T_{\mathrm{n}}$ are given in Table II.

The $T_{\mathrm{n}}{ }^{\prime}$ values were very different for the different spin probes. As found for $T_{50 \mathrm{G}}, T_{\mathrm{n}}{ }^{\prime}$ decreased with increasing methylene chain length, but the effects of drawing were not found for SPIV and SPV. $T_{\mathrm{n}}{ }^{\prime}$ decreased in the order of SPIII $>$ SPV $>$ SPIV, suggesting that the rotation of a piperidine ring becomes easier in this order. The difference between the $T_{\mathrm{n}}{ }^{\prime}$ values for NY-4 and NY-12 was 34,52 , and $77 \mathrm{~K}$ for SPIII, SPV, and SPIV, respectively. This indicates that the more easily the rotaion of a piperidine ring occurs, the more sensitively $T_{\mathrm{n}}{ }^{\prime}$ reflects the effects of the methylene chain length. Thus $T_{\mathrm{n}}{ }^{\prime}$ is found to be a good measure to estimate the effects of a functional group in a spin

Table II. $T_{\mathrm{n}}{ }^{\prime}$ and $T_{\mathrm{n}}(\mathrm{K})$

\begin{tabular}{|c|c|c|c|c|c|c|}
\hline & Substituent & NY-4 & NY-6(2D) & NY-6(ND) & NY-11 & $N Y-12$ \\
\hline & \multicolumn{6}{|c|}{$T_{\mathrm{n}}^{\prime}$} \\
\hline SPIII $^{\mathrm{a}}$ & $=\mathrm{NH}_{2}$ & 366 & 365 & 347 & 333 & 332 \\
\hline SPIV & None & 314 & 290 & 288 & 252 & 237 \\
\hline \multirow[t]{2}{*}{ SPV } & $-\mathrm{OH}$ & 345 & 330 & 326 & 302 & 293 \\
\hline & \multicolumn{6}{|c|}{$T_{\mathrm{n}}$} \\
\hline SPIII $^{\mathrm{a}}$ & $-\mathrm{NH}_{2}$ & & & 403 & 379 & 380 \\
\hline SPIV & None & 398 & 390 & 386 & 375 & 365 \\
\hline SPV & $-\mathrm{OH}$ & 406 & 395 & 392 & 382 & 372 \\
\hline SPVI & $=\mathrm{O}$ & 405 & 389 & 394 & 366 & 359 \\
\hline
\end{tabular}

a $C f$. ref 13.

Table III. $E_{\mathrm{a}}^{\mathrm{R}}\left(\mathrm{kJ} \mathrm{mol}^{-1}\right)$

\begin{tabular}{|c|c|c|c|c|c|c|}
\hline & Substituent & NY-4 & NY-6(2D) & NY-6(ND) & NY-11 & NY-12 \\
\hline \multicolumn{7}{|c|}{ "low" } \\
\hline SPIII $^{\mathrm{a}}$ & $-\mathrm{NH}_{2}$ & $7.9 \pm 0.4$ & $6.8 \pm 0.3$ & $6.77 \pm 0.09$ & $6.6 \pm 0.3$ & $5.69 \pm 0.13$ \\
\hline SPIV & None & $4.19 \pm 0.13$ & $5.3 \pm 0.2$ & $5.67 \pm 0.13$ & $6.0 \pm 0.5$ & $4.4 \pm 0.4$ \\
\hline SPV & $-\mathrm{OH}$ & $6.59 \pm 0.18$ & $5.7 \pm 0.2$ & $4.9 \pm 0.3$ & $5.7 \pm 0.3$ & $4.9 \pm 0.3$ \\
\hline SPVI & $=\mathrm{O}$ & $6.62 \pm 0.14$ & $5.92 \pm 0.18$ & $6.00 \pm 0.16$ & $4.98 \pm 0.12$ & $5.26 \pm 0.12$ \\
\hline \multicolumn{7}{|c|}{ "medium" } \\
\hline SPIII $^{\mathrm{a}}$ & $-\mathrm{NH}_{2}$ & $26.1 \pm 1.2$ & $47.5 \pm 1.3$ & $35.5 \pm 0.9$ & $32 \pm 2$ & $26 \pm 2$ \\
\hline SPIV & None & $15.1 \pm 0.5$ & $11.1 \pm 0.8$ & $11.6 \pm 0.8$ & $8.92 \pm 0.13$ & $7.7 \pm 0.2$ \\
\hline SPV & $-\mathrm{OH}$ & $25.0 \pm 1.1$ & $24 \pm 2$ & $24.0 \pm 0.8$ & $15.1 \pm 1.1$ & $14.9 \pm 0.9$ \\
\hline \multicolumn{7}{|c|}{ "high" } \\
\hline SPIII $^{a}$ & $-\mathrm{NH}_{2}$ & & & $53.2 \pm 1.7$ & $68 \pm 3$ & $73 \pm 4$ \\
\hline SPIV & None & $53.8 \pm 0.6$ & $64 \pm 2$ & $60.3 \pm 1.4$ & $69 \pm 4$ & $67.5 \pm 1.9$ \\
\hline SPV & $-\mathrm{OH}$ & $56.2 \pm 1.1$ & $62.3 \pm 1.5$ & $61.6 \pm 1.9$ & $73 \pm 2$ & $71 \pm 2$ \\
\hline SPVI & $=\mathrm{O}$ & $56 \pm 2$ & $65 \pm 5$ & $56.0 \pm 1.8$ & $54.5 \pm 1.6$ & $62 \pm 4$ \\
\hline
\end{tabular}

a $C f$. ref 13. 
probe.

$T_{\mathrm{n}}$ scarcely varied from probe to probe, and was dependent on only the methylene chain length of the nylon. From this, it is thought that at $T_{\mathrm{n}}{ }^{\prime}$ the hydrogen bonds between the spin probes and the nylon chains are broken, and the spin probes start to diffuse translationally. An azo dye type spin probe, SPI, has a larger molecular volume than the spin probes used here, and its $T_{\mathrm{n}}$ was about $30 \mathrm{~K}$ hihger. ${ }^{13}$ In addition, an anionic spin probe containing a carboxylate group, whose molecular volume is similar to the spin probes used here, began to diffuse translationally at a temperature $30 \mathrm{~K}$ higher. ${ }^{15}$ (In ref 15 this temperature is designated as $T_{\mathrm{n}}{ }^{\prime \prime}$.) In this case the anionic spin probe is thought to interact with the amino end group of the nylon below $T_{\mathrm{n}}{ }^{\prime \prime}$, but the electrostatic interaction is broken at $T_{\mathrm{n}}{ }^{\prime \prime}$. From the above results, we can conclude that $T_{\mathrm{n}}$ depends not only on the molecular volumes of spin probes, but also on the strength of the interactions between the probe molecules and the polymer chains.

The activation energies for rotation $E_{\mathrm{a}}{ }^{\mathbf{R}}$ were determined from the Arrhenius plots of $\tau_{\mathrm{R}}$ and are given in Table III. $E_{\mathrm{a}}{ }^{\mathrm{R}}$ in the "low" temperature region below $T_{\mathrm{n}}$ ' (for SPVI, below $T_{n}$ ) was of the same order for all the systems. The spin probes probably undergo rotational vibrations in this region. $E_{\mathrm{a}} \mathbf{R}$ in the "medium" temperature region between $T_{\mathrm{n}}$ ' and $T_{\mathrm{n}}$ varied from system to system. For SPIV and SPV, no effect of drawing on $E_{\mathrm{a}}{ }^{\mathbf{R}}$ in this region could be found. Only for SPIII could effects of drawing be found, presumably owing to the peculiar behavior of the amino group of SPIII, ${ }^{13}$ as mentioned in the discussions of $T_{50 \mathrm{G}}$ and $T_{\mathrm{n}}{ }^{\prime}$. The coupled effects of the methylene chain length and of drawing made the behavior of $E_{\mathrm{a}}{ }^{\mathrm{R}}$ for SPIII very complicated, whereas $E_{\mathrm{a}}{ }^{\mathrm{R}}$ for SPIV and SPV decreased monotonically with increasing methylene chain length of the nylon. This suggests that the longer methylene chains make it easier to form local free volume for the rotation of a piperidine ring. Also $E_{\mathrm{a}}{ }^{\mathrm{R}}$ in the "medium" temperature region decreased in the order of SPIII $>$ SPV $>$ SPIV, supporting the idea that the restriction becomes weaker in this order.

$E_{\mathrm{a}}^{\mathrm{R}}$ in the "high" temperature region increased with increasing methylene chain length for SPIII, SPIV, and SPV, while remaining almost constant for SPVI. This result is opposite to that in the "medium" temperature region, and can not be interpreted by considering only the rotational motion of the spin probes. Here we should also consider the translational diffusion of the probe molecules. As was mentioned in our previous paper, ${ }^{13}$ the activation energy of translational diffusion is affected by a jumping distance and a barrier height. The molecular motion of the nylon chains becomes greater with an increase in the number of methylene groups, and the jumping distance becomes larger, resulting in the larger $E_{\mathrm{a}}{ }^{\mathrm{R}}$. For SPIII, SPIV, and SPV, the jumping distance and the barrier height are thought to be similar, because their activation energies were similar. Thus the consideration of the translational diffusion make it possible to interpret reasonably the results in the "high" temperature region. On the other hand, $E_{\mathrm{a}}{ }^{\mathrm{R}}$ for SPVI in this region was unusual. The reason for this is not clear, and it is dangerous to conclude that the carbonyl group alone leads to this result.

\section{Anisotropy or Rotation}

The anisotropy parameter $\varepsilon$ was calculated from the ESR spectra, as described in our previous paper. ${ }^{13} \varepsilon$ reflects only the anisotropy of motion in the region of $\tau_{\mathrm{R}}<1 \times 10^{-9} \mathrm{~s}$, but it also includes the mobility of the probe molecules in the region of $\tau_{\mathrm{R}}>1 \times 10^{-9} \mathrm{~s}$, so that $\varepsilon$ in this latter region cannot be used for this discussion. Figure 6 shows the change of $\varepsilon$ with temperature for NY-12. The behavior of $\varepsilon$ for the other nylons was a similar pattern to that for NY-12. The $\varepsilon$ values for SPIV and SPVI became close to zero in the region of 


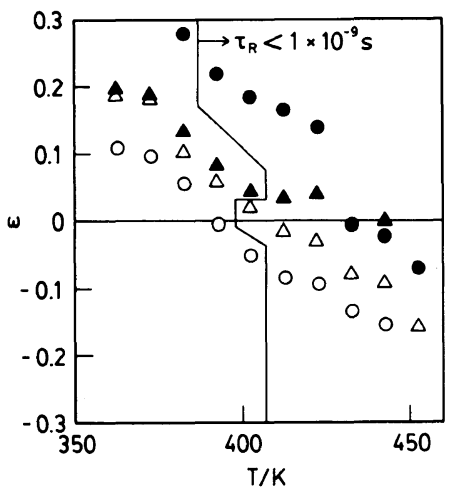

Figure 6. $\varepsilon$ vs. temperature for $\mathrm{NY}-12$. $\bigcirc$, SPIII*(-NH $\left.{ }_{2}\right) ; 0, \operatorname{SPIV}($ None $) ; \Delta, \operatorname{SPV}(-\mathrm{OH}) ; \boldsymbol{\Delta}$, $\operatorname{SPVI}(=0) .{ }^{*} C f$. ref 13 .

$\tau_{\mathbf{R}}<1 \times 10^{-9} \mathrm{~s}$, while those for SPIII and SPV became negative in this region. This suggests that SPIV and SPVI rotate isotropically, and SPIII and SPV rotate anisotropically around the $x$ axis, ${ }^{18}$ which corresponds to the $\mathrm{N}-\mathrm{O}$ bond direction. However, the degree of the anisotropy was rather small, considering the absolute values of $\varepsilon$. Such slight difference probably comes from the fine structures of the probe molecules. It is thus thought that the spin probes having an amino or a hydroxyl group rotate anisotropically around the $x$ axis, because the existence of these functional groups makes the $x$ axis to be a long axis around which to rotate more easily.

\section{CONCLUSION}

From the above results, we can conclude that the mobilities of the spin probes in the nylon films are influenced by the functional groups in the probe molecules, owing to the interactions between the functional groups and the nylon chains. It was suggested in our previous study ${ }^{13}$ that an amino group in a spin probe formed a crosslinkage through two hydrogen bonds, while spin probes with a hydroxyl or a carbonyl group did not form such a crosslinkage. As a result, only for SPIII the effects of drawing could be observed. Also the single bonds in the spin probes affected their mobilities. This effect was observed mainly in the "medium" temperature region, where the rotation of a piperidine ring probably occurs without extensive translational diffusion. In the "high" 'temperature region, the hydrogen bonds were probably broken, and the effects of the functional groups were very small. It is thus concluded that the interactions between probe molecules and polymer chains are a main factor influencing the mobilities of the probe molecules in the temperature ranges where these interactions are maintained, and where extensive rotational motions of the piperidine ring occur.

Acknowledgment. We express hearty thanks to Prof. Dr. Hiroshi Tomiyasu and Dr. Masayuki Harada of Research Laboratory for Nuclear Reactors, Tokyo Institute of Technology, for ESR measurements. The award in 1986 of a visiting professorship to $\mathrm{R}$. McG. by Ministry of Education of Japan is gratefully acknowledged.

\section{REFERENCES}

1. M. H. Cohen and D. Turnbull, J. Chem. Phys., 31, 1164 (1959).

2. H. Yasuda and C. E. Lamaze, J. Macromol. Sci., B5, 111 (1971).

3. S. Wisniewski and S. W. Kim, J. Membrane Sci., 6, 299 (1980).

4. N. A. Peppas and C. T. Reinhart, J. Membrane Sci., 15, 275 (1983).

5. C. T. Reinhart and N. A. Peppas, J. Membrane Sci., 18, 227 (1984).

6. e.g., Y. Kimura, H.-J. Lim, and T. Iijima, Angew. Makromol. Chem., 138, 151 (1986).

7. e.g., K. Kimura, M. Yoshinaga, S. Kitazawa, and T. Shono, J. Polym. Sci., Polym. Chem. Ed., 21, 2777 (1983).

8. N. Kusumoto, S. Sano, N. Zaitsu, and Y. Motozato, Polymer, 17, 448 (1976).

9. A. T. Bullock, G. G. Cameron, and I. S. Miles, Polymer, 23, 1536 (1982).

10. Z. Hlouskova, J. Tino, and E. Borsig, Polym. Commun., 25, 112 (1984).

11. F. Bueche, Ed., "Physical Properties of Polymers," Wiley-Interscience, New York, 1962.

12. K. Hamada, T. Iijima, and R. McGregor, Polym. J., 


\section{Effects of Hydrogen Bonds on the Mobility}

17, 1245 (1985).

13. K. Hamada, T. Iijima, and R. McGregor, Macromolecules, 19, 1443 (1986).

14. K. Hamada, T. Iijima, and R. McGregor, J. Polym. Sci., Polym. Phys. Ed., in press.

15. K. Hamada, T. Iijima, and R. McGregor, Macromolecules, 20, 215 (1987).
16. D. Kivelson, J. Chem. Phys., 33, 1094 (1960).

17. A. N. Kuznetsov, A. M. Vasserman, A. U. Volkov, and N. N. Korst, Chem. Phys. Lett., 12, 103 (1971).

18. O. H. Griffith and P. C. Jost, "Spin Labeling. Theory and Applications," L. J. Berliner, Ed., Academic Press, New York, 1976, Vol. 1, p 453. 